\title{
Efeito do treinamento de força sobre a capacidade funcional de idosos ativos: uma revisão sistemática
}

\author{
Effect of strength training on the functional capacity of active elderly: systematic review \\ Efecto del entrenamiento de fuerza sobre la capacidad funcional de ancianos activos: revisión \\ sistemática
}

Recebido: 02/09/2021 | Revisado: 09/09/2021 | Aceito: 10/09/2021 | Publicado: 13/09/2021

\author{
Raquel Conceição da Silva \\ ORCID: https://orcid.org/0000-0002-9705-523X \\ Centro Universitário UniREDENTOR, Brasil \\ E-mail: raqsilva23@gmail.com \\ Estêvão Rios Monteiro \\ ORCID: https://orcid.org/0000-0003-1866-553X \\ Centro Universitário Augusto Motta, Brasil \\ Universidade Federal do Rio de Janeiro, Brasil \\ Instituto Brasileiro de Medicina e Reabilitação, Brasil \\ E-mail: profestevaomonteiro@gmail.com \\ Rafael Carvalho da Silva Mocarzel \\ ORCID: https://orcid.org/0000-0001-9480-826X \\ Universidade de Vassouras, Brasil \\ Universidade Federal do Rio de Janeiro, Brasil \\ Universidade do Porto, Portugal \\ E-mail: professormocarzel@gmail.com
}

\begin{abstract}
Resumo
O objetivo do presente estudo foi revisar sistematicamente o efeito do treinamento de força a capacidade funcional de idosos ativos. A pesquisa foi realizada nas bases indexadoras Scielo, MedLine e Lilacs, assim como nos repositórios da PEDro e Google Acadêmico entre os anos de 2010 e 2020. Os seguintes descritores foram utilizados para busca em diferentes combinações: "exercício resistido" ou "treinamento de força", e "idosos" ou "envelhecimento" e "capacidade funcional" tendo suas respectivas traduções para o inglês 'strength training' or 'resistance training' and 'elderly' and 'functional capacity'. No total 14 artigos foram incluídos na revisão, onde todos eles apresentaram resultados de melhora na capacidade funcional em idosos após intervenção com treinamento de força. Os protocolos de treinamento de força como forma de intervenção numa população idosa e ativa são capazes de melhorar a capacidade funcional através do aumento na massa e força muscular, densidade mineral óssea e capacidade funcional. Conclui-se com base na presente revisão que o treinamento de força é capaz de melhorar a capacidade funcional de idosos. Além disso, a proposta de treinamento demonstrou otimização do equilíbrio, marcha, densidade mineral óssea e da massa muscular de indivíduos idosos.
\end{abstract}

Palavras-chave: Envelhecimento; Exercício físico; Exercício resistido; Funcionalidade; Revisão sistemática.

\begin{abstract}
The study purpose was to systematically review the effect of strength training on the active elderly functional capacity. The search was carried out in the Scielo, MedLine and Lilacs indexing databases, as well as in the PEDro and Google Academic repositories between 2010 and 2020. The following descriptors were used to search in different combinations: 'strength training' or 'resistance training' and 'elderly' and 'functional capacity'. Fourteen studies were included in the present review, which showed improvement in the functional capacity of elderly people after strength training intervention. Strength training protocols as a form of intervention in an elderly and active population are able to improve functional capacity by increasing muscle mass and strength, bone mineral density and functional capacity. It is concluded that strength training is able to improve the functional capacity of elderly people. In addition, the training proposal demonstrated optimization of balance, gait, bone mineral density and muscle mass in elderly individuals.
\end{abstract}

Keywords: Elderly; Physical exercise; Resistance exercise; Functionality; Systematic review.

\section{Resumen}

El propósito del estudio fue revisar sistemáticamente el efecto del entrenamiento de fuerza sobre la capacidad funcional activa del anciano. La búsqueda se realizó en las bases de datos de indexación Scielo, MedLine y Lilacs, así como en los repositorios PEDro y Google Academic entre 2010 y 2020. Se utilizaron los siguientes descriptores para buscar en diferentes combinaciones: "entrenamiento de fuerza" o "entrenamiento de resistencia" y "ancianos" y 
"capacidad funcional". Se incluyeron catorce estudios en la presente revisión, que mostraron una mejora en la capacidad funcional de los ancianos después de la intervención de entrenamiento de fuerza. Los protocolos de entrenamiento de fuerza como forma de intervención en una población anciana y activa son capaces de mejorar la capacidad funcional aumentando la masa y fuerza muscular, la densidad mineral ósea y la capacidad funcional. Se concluye que el entrenamiento de fuerza es capaz de mejorar la capacidad funcional de las personas mayores. Además, la propuesta de entrenamiento demostró la optimización del equilibrio, la marcha, la densidad mineral ósea y la masa muscular en personas mayores.

Palabras clave: Anciano; Ejercicio físico; Ejercicio de resistencia; Funcionalidad; Revisión sistemática.

\section{Introdução}

Notoriamente a população mundial está envelhecendo e continuará a envelhecer, onde se calcula que até 2050 haverá dois bilhões de indivíduos com idade mínima de 60 anos em todo o mundo (Moreira e Henriques, 2014). Sobre o envelhecimento, não é equivocado dizer que os adoecimentos, inatividade, depressão, solidão e inabilidades são algumas das representações distorcidas associadas às etapas mais avançadas da vida (Lima, 2010). Desta forma, falar de envelhecimento é abrir uma ampla gama de entendimentos que tecem ao cotidiano e as diferentes experiências culturais que as pessoas experimentaram em vida. Mudanças fisiológicas e alterações funcionais são fatores constantes na vida daqueles que enfrentam o envelhecimento. Por isso, para manter-se ativo e desfrutar de uma velhice saudável, sugere-se uma vida com independência funcional, ocupação, habilidade e descontração (Silva e Santos, 2010; Oliveira e Menezes, 2011). Se tratando do idoso, a prática das atividades básicas mostra-se relevante para a sua sobrevivência (Oliveira e Menezes, 2011). A degradação do processo natural do envelhecimento favorece negativamente para perda de função dessa população (Carvalho et al., 2017). A capacidade funcional constitui uma integração sensória motora que engloba coordenação, equilíbrio e marcha. Essa falta funcionalidade, além da perda de massa muscular, equilíbrio e coordenação, faz com que o idoso esteja propenso a outras doenças (Rejeski et al., 2012). Além disso, atua favorecendo a autonomia do idoso na saúde física e mental (Agner et al., 2013).

Praticantes de exercícios regulares dormem melhor, possuem mais disposição para realizar as atividades cotidianas, tornam-se menos propício às enfermidades, envelhecem de autônoma (Cipriano, 2010; Rodrigues, 2014). Sendo assim, é recomendada a prática do treinamento de força, pois traz benefícios ao sistema músculo esquelético e endócrino, com a evolução do padrão de ativação das fibras musculares e unidades motoras (Leenders et al., 2013). Conforme os estudos citados, o treinamento de força é o mais adequado para o idoso em virtude de ganhar mais flexibilidade (Helriigle et al., 2013), equilíbrio (Elias et al., 2012) e capacidade funcional (Moura et al., 2012). A resistência exigida ao músculo pode ser gerada pelo peso corporal, pesos livres ou aparelhos que promovam alguma resistência (Câmara e Ester, 2012). Considerando a importância da potência muscular para os idosos, o treinamento de força é aplicado com o objetivo de desacelerar a baixa diminuição de força, potência e massa muscular, com a finalidade de preservar a capacidade funcional e qualidade de vida do idoso (Cipriani et al., 2010). Estudos apontam que o treinamento de força influência de forma positiva na musculatura esquelética (Marques, Mota e Carvalho, 2012). Por esta razão, os idosos cada vez mais são motivados a envolver-se na prática do treinamento de força (ACSM, 2011).

Este estudo torna-se relevante por demostrar através de uma revisão bibliográfica, o efeito do treinamento de força na capacidade funcional de idosos ativos, podendo ser destacado como uma possível ferramenta de preparação física e reabilitação mioarticular capaz de diminuir e retardar a debilidade inúmeros fatores provocados pela velhice. Além disso, a prática do treinamento de força para idosos não só é importante para capacidade funcional, mas um bem essencial para evitar a prostração e a solidão, sendo assim, o idoso é beneficiado como um todo ao incluir nas suas atividades físicas o treinamento de força. Diante disso, o objetivo do presente estudo foi revisar o efeito do treinamento de força na capacidade funcional de idosos ativos. 


\section{Metodologia}

\subsection{Tipo de estudo}

O estudo em tela é uma pesquisa de cunho de revisão de literatura (Estrela, 2018).

\subsection{Seleção dos artigos}

A pesquisa foi realizada nas bases indexadoras Scielo, MedLine e Lilacs, assim como nos repositórios da PEDro e Google Acadêmico entre os anos de 2010 e 2020. Os seguintes descritores foram utilizados para busca em diferentes combinações: "exercício resistido" ou "treinamento de força", e "idosos" ou "envelhecimento" e "capacidade funcional" tendo suas respectivas traduções para o inglês 'strength training' or 'resistance training' and 'elderly' and 'functional capacity'.

Os estudos encontrados foram selecionados dentro dos seguintes critérios de inclusão: a) ser um artigo original, b) estar redigido na língua portuguesa ou inglesa, c) idosos fisicamente ativos e saudáveis e d) artigos que comparassem o treinamento de força com outra modalidade de exercícios. Foram excluídos artigos que investigavam sujeitos em treinamento sob a ação de recursos ergogênicos, e estudos realizados em animais.

Dois pesquisadores leram os resumos dos artigos encontrados na busca e aplicaram os critérios de inclusão e exclusão pertinentes. Após a pré-seleção feita por cada um, eles se encontraram e os artigos congruentemente inseridos ou retirados da composição da presente revisão foram assim destinados. Quando houve desacordo entre as decisões dos dois pesquisadores, ambos discutiram exaustivamente o estudo até chegar a uma decisão de comum acordo. Quando não se encontrou uma decisão acordada, um terceiro pesquisador foi consultado a respeito do estudo. Os artigos em que os resumos atenderam aos critérios de seleção foram então lidos na íntegra e as informações extraídas baseada na estratégia de PICOT (População, Comparação, Resultados e Desenho do Estudo).

\section{Resultados e Discussão}

$\mathrm{Na}$ busca por artigos significativos para a pesquisa, foram recuperados 20 artigos, 05 desses artigos foram excluídos por não atenderem o critério de inclusão do presente estudo (ver Quadro 1). Dos 15 estudos avaliados, 09 encontraram melhora da mobilidade funcional. Os estudos incluíram rotinas de exercícios resistidos variando de 12 a 15 semanas de intervenção. A frequência semanal variou de 2 a 3 vezes por semana. 
Quadro 1 - Descrição dos estudos selecionados.

\begin{tabular}{|c|c|c|c|c|c|c|}
\hline Autores e ano & População & Intervenção & Comparação & Protocolo & Resultados & Conclusão \\
\hline $\begin{array}{l}\text { Cipriane } \text { et al. } \\
\text { (2010) }\end{array}$ & $\begin{array}{l}\text { Estudo realizado com } 225 \\
\text { idosos, média de idade de } \\
69,26 \text { anos }(\mathrm{dp}=5,685) .\end{array}$ & 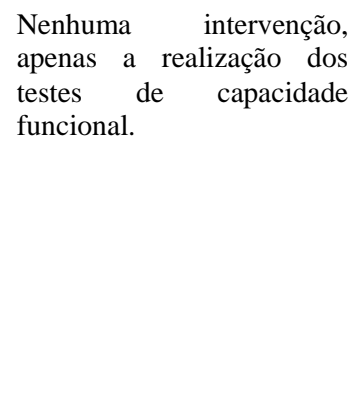 & 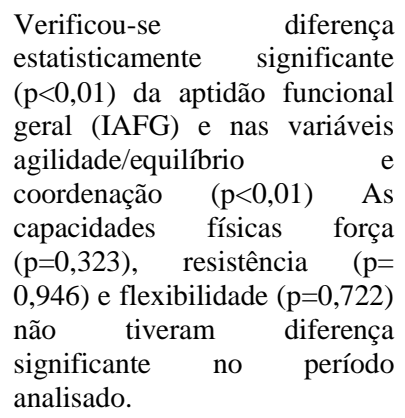 & 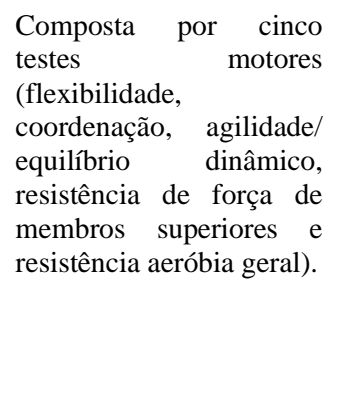 & $\begin{array}{l}\text { Verificou-se também que o } \\
\text { grupo que praticava } \\
\text { atividades físicas há mais de } \\
10 \text { anos e com faixa etária } \\
\text { acima de } 80 \text { anos de idade, } \\
\text { apresentaram melhor índice } \\
\text { de IAFG nas avaliações } \\
\text { realizadas. }\end{array}$ & $\begin{array}{l}\text { Conclui-se que a prática de } \\
\text { atividades físicas por idosos } \\
\text { pode ser um importante fator } \\
\text { para a melhora e/ou } \\
\text { manutenção da aptidão } \\
\text { funcional no decorrer do } \\
\text { processo de envelhecimento. }\end{array}$ \\
\hline Liu e Latham (2011) & $\begin{array}{l}\text { Participaram do estudo, } \\
\text { idosas de } 65 \text { a } 80 \text { anos, com } \\
\mathrm{IMC} \geq 30 \mathrm{~kg} / \mathrm{m}^{2} \text { e força de } \\
\text { preensão palmar (FPP) } \leq 21 \\
\text { Kgf. }\end{array}$ & $\begin{array}{l}\text { GES: grupo de exercícios - } \\
10 \text { semanas de treinamento } \\
\text { resistido para força, } \\
\text { potência e resistência. } \\
\text { GC: grupo controle - sem } \\
\text { nenhuma intervenção - } \\
\text { recebeu ligações telefônicas } \\
\text { semanais. }\end{array}$ & $\begin{array}{l}\mathrm{Na} 1^{\mathrm{a}} \text { semana, os grupos } \\
\text { apresentaram características } \\
\text { similares. Após a intervenção, o } \\
\text { GES apresentou ganhos de } 20 \% \\
\text { na potência de extensores de } \\
\text { joelho }(p<0,001, r=-0,55) \mathrm{e} \\
10 \% \text { na FPP }(p<0,001, r=- \\
0,66) \text {. Não houve diferença nos } \\
\text { outros níveis de funcionalidade. } \\
\mathrm{O} \text { número necessário a tratar } \\
\text { (NNT) para velocidade de } \\
\text { marcha foi de } 5 \text { pacientes. O } \\
\text { grupo de exercícios (GES) } \\
\text { apresentou diferença } \\
\text { significativa para o } \\
\text { desempenho muscular }(p= \\
0,05, r=-0,36) \text { Não houve } \\
\text { diferença no desempenho } \\
\text { funcional }(p=0,74, r=-033) .\end{array}$ & $\begin{array}{l}\text { Foram realizados os } \\
\text { testes: Short Physical } \\
\text { Performance Battery } \\
\text { (SPPB), velocidade usual } \\
(10 \text { m) e máxima de } \\
\text { marcha (Shuttle Walking } \\
\text { Test }) \text {, velocidade usual } \\
\text { para subir e descer } \\
\text { escadas e máxima para } \\
\text { subir escadas. }\end{array}$ & $\begin{array}{l}\text { A análise final contou com } 14 \\
\text { idosas em cada grupo. O GES } \\
\text { apresentou ganhos de } 20 \% \text { na } \\
\text { potência muscular }(p<0,001 \text {, } \\
r=-0,55) \text { e } 10 \% \text { na FPP } \\
\text { (p<0,001, } r=-0,66 \text { ). Não } \\
\text { foram observadas diferenças } \\
\text { entre os grupos nos níveis de } \\
\text { atividades e participação. }\end{array}$ & $\begin{array}{l}\text { Conclui-se que idosas com } \\
\text { OS podem se beneficiar de } \\
\text { exercícios resistidos para a } \\
\text { melhora na função muscular. } \\
\text { O baixo NNT para melhoras } \\
\text { na marcha reforça a } \\
\text { aplicabilidade clínica desta } \\
\text { intervenção. }\end{array}$ \\
\hline Orsatti et al. (2011) & $\begin{array}{l}\text { Foram selecionadas } 52 \\
\text { mulheres, sedentárias, com } \\
\text { idade entre } 40 \text { e } 70 \text { anos. }\end{array}$ & $\begin{array}{l}\text { Avaliações antropométricas } \\
\text { e de composição corporal. } \\
\text { Após, foram agendados os } \\
\text { dias de familiarização com } \\
\text { o equipamento de } \\
\text { musculação e o teste de } \\
\text { uma repetição máxima } \\
\text { (1RM). No último dia de } \\
\text { familiarização, foi realizada } \\
\text { a estimativa de força }\end{array}$ & $\begin{array}{l}\text { A qualidade muscular parece } \\
\text { não se alterar em mulheres } \\
\text { entre } 40 \text { e } 60 \text { anos, quando } \\
\text { comparadas por grupos etários. }\end{array}$ & $\begin{array}{l}\text { As participantes } \\
\text { realizaram três sessões de } \\
\text { exercícios, em dias } \\
\text { alternados, A força dos } \\
\text { grupamentos musculares } \\
\text { foi testada nos exercícios } \\
\text { de rosca direta, cadeira } \\
\text { extensora e supino reto. } \\
\text { Estipulou-se } \\
\text { subjetiva parga } \\
\text { a o }\end{array}$ & 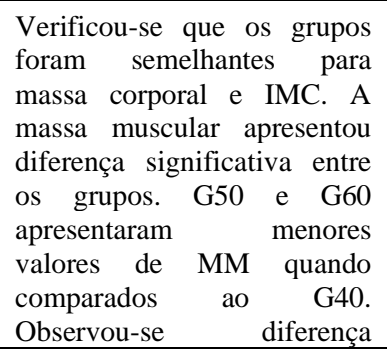 & $\begin{array}{l}\text { O presente estudo mostrou } \\
\text { que a qualidade muscular } \\
\text { parece não se alterar em } \\
\text { mulheres entre } 40 \text { e } 60 \text { anos, } \\
\text { quando comparadas por } \\
\text { grupos etários. Assim, a } \\
\text { redução da massa muscular } \\
\text { parece ser importante fator } \\
\text { para os valores menores de } \\
\text { força muscular em mulheres }\end{array}$ \\
\hline
\end{tabular}


Research, Society and Development, v. 10, n. 12, e47101220148, 2021

(CC BY 4.0) | ISSN 2525-3409 | DOI: http://dx.doi.org/10.33448/rsd-v10i12.20148

\begin{tabular}{|c|c|c|c|c|c|c|}
\hline & & $\begin{array}{l}\text { máxima }(\mathrm{EFM}) \\
\text { estimar a } \% \text { de cara } \\
\text { necessária para realização } \\
\text { do teste de uma repetição } \\
\text { máxima (1RM), realizado } \\
\text { dois dias após o EFM. }\end{array}$ & & $\begin{array}{l}\text { aquecimento, realizando } \\
\text { entre } 10 \text { - } 15 \text { repetições } \\
\text { com cargas que } \\
\text { representassem de } 40 \% \text { a } \\
60 \% \text { da máxima estimada } \\
\text { pelo EFM. }\end{array}$ & $\begin{array}{l}\text { percentual de } 10,1 \% \text {, } \\
\text { significativa na MM entre } \\
\text { G40 e G50 e de } 7,5 \% \text { entre } \\
\text { G50 e G60, totalizando-se } \\
16,8 \% \text { entre G40 e G60. }\end{array}$ & entre 40 e 60 anos. \\
\hline Elias et al. (2012) & $\begin{array}{l}\text { Constituída por } 18 \\
\text { mulheres idosas, com idade } \\
\text { média de } 65,5 \pm 5,84 \\
\text { praticantes das aulas de } \\
\text { hidroginástica do Centro de } \\
\text { Excelência em Atividade } \\
\text { Física - CEAF. }\end{array}$ & $\begin{array}{l}\text { A aptidão física foi } \\
\text { avaliada através da bateria } \\
\text { de testes de Rikli e Jones, } \\
\text { com avaliação de força de } \\
\text { membros inferiores, força } \\
\text { de membros superiores, } \\
\text { flexibilidade dos membros } \\
\text { inferiores, mobilidade } \\
\text { física (potência, velocidade, } \\
\text { agilidade e equilíbrio), } \\
\text { flexibilidade dos membros } \\
\text { superiores, resistência } \\
\text { aeróbia e índice de massa } \\
\text { corporal. }\end{array}$ & $\begin{array}{l}\text { Os avaliados atingiram bons } \\
\text { níveis de aptidão física nos } \\
\text { testes de força de membros } \\
\text { superior, flexibilidade de } \\
\text { membros inferiores e } \\
\text { mobilidade física. Já nos testes } \\
\text { de força de membro inferior, } \\
\text { capacidade aeróbia e e } \\
\text { flexibilidade de membros } \\
\text { superiores, os níveis de aptidão } \\
\text { física foram baixos. }\end{array}$ & $\begin{array}{l}\text { Aulas ministradas no } \\
\text { período da tarde numa } \\
\text { piscina com profundidade } \\
\text { de } 1,10 \mathrm{~m} \text {, medindo } 25 \mathrm{~m} \\
\text { x } 12,5 \mathrm{~m} \text {, com água na } \\
\text { temperatura aproximada } \\
\text { de } 26 \text { a } 28^{\circ} \mathrm{C} \text {. As aulas } \\
\text { consistiam em quatro } \\
\text { fases: } 1 \text { - Aquecimento } \\
\text { (alongamento } \\
\text { flexibilidade, método } \\
\text { estático, durante } 5 \text { min); } \\
2 \text { - Exercícios aeróbicos } \\
\text { (corridas, deslocamentos } \\
\text { e movimentos } \\
\text { combinados de braços e } \\
\text { pernas; } 3 \text { - Exercícios } \\
\text { localizados } \\
\text { (força/resistência dos } \\
\text { membros superiores, } \\
\text { inferiores e abdominais, } \\
\text { utilizando a resistência da } \\
\text { água, 4 - Relaxamento } \\
\text { (caminhadas lentas, por } 5 \\
\text { min). }\end{array}$ & $\begin{array}{l}\text { Sessenta e cinco por cento da } \\
\text { amostra apresentaram } \\
\text { excesso de peso e obtiveram } \\
\text { resultados inferiores na } \\
\text { maioria dos testes de aptidão } \\
\text { física funcional. }\end{array}$ & $\begin{array}{l}\text { Conclui-se que os idosos } \\
\text { praticantes de aulas de } \\
\text { hidroginástica } \\
\text { apresentaram boa aptidão } \\
\text { física funcional geral, } \\
\text { principalmente nos níveis de } \\
\text { força muscular de membro } \\
\text { inferior, desempenho } \\
\text { cardiorrespiratório } \\
\text { flexibilidade. }\end{array}$ \\
\hline $\begin{array}{l}\text { Granacher } \text { et al. } \\
\text { (2012) }\end{array}$ & $\begin{array}{l}36 \text { idosos ativos. } \\
\text { G1: } \mathrm{n}=18 \text { e idade }=69,4 \pm \\
6,8 \text { anos. } \\
\text { G2: } \mathrm{n}=18 ; 69,4 \pm 5,7 \text { anos. }\end{array}$ & $\begin{array}{l}\text { G1: realizou Teste sentar } \\
\text { levantar com 5 repetições } \\
\text { (STS-5), Teste de } \\
\text { Caminhada de seis metros } \\
\text { (SMW) e Teste de Alcance } \\
\text { funcional (FRT). } \\
\text { G2: realizou Teste sentar- } \\
\text { levantar com } 5 \text { repetições } \\
\text { (STS-5), Teste de } \\
\text { Caminhada de seis metros } \\
\text { (SMW) e Teste de Alcance } \\
\text { funcional (FRT). }\end{array}$ & $\begin{array}{l}\text { Foi realizada a comparação } \\
\text { entre o G1 e G2 para verificar } \\
\text { os efeitos do treinamento de } \\
\text { força, de equilíbrio e } \\
\text { alongamentos sobre a } \\
\text { capacidade funcional de idosas } \\
\text { com baixa massa óssea. } \\
\text { Capacidade funcional: foi } \\
\text { realizada através dos seguintes } \\
\text { testes de mobilidade funcional: } \\
\text { teste de sentar-levantar com } 5 \\
\text { repetições no Sit-to-Stand test, } \\
\text { o teste de caminhada de } 6 \\
\text { metros- Six-metre-walk e o teste }\end{array}$ & $\begin{array}{l}\text { G1: realizou durante } 4 \\
\text { meses, 2 vezes por } \\
\text { semana, com treina- } \\
\text { mento de força muscular } \\
\text { e mobilidade funcional. } \\
\text { G2: realizou } \\
\text { alongamentos e palestras } \\
\text { informativas. }\end{array}$ & $\begin{array}{l}\text { Indivíduos que realizaram } \\
\text { fortalecimento de } \\
\text { musculatura estabilizadora do } \\
\text { tronco obtiveram melhora na } \\
\text { sua capacidade funcional. }\end{array}$ & $\begin{array}{l}\text { Concluiu-se que houve } \\
\text { melhoras em ambos os } \\
\text { grupos estudados e que os } \\
\text { exercícios físicos propostos } \\
\text { são capazes de trazer } \\
\text { benefícios para idosas com } \\
\text { baixa massa óssea, através de } \\
\text { melhora na sua capacidade } \\
\text { funcional. }\end{array}$ \\
\hline
\end{tabular}




\begin{tabular}{|c|c|c|c|c|c|c|}
\hline & & & $\begin{array}{l}\text { de alcance funcional } \\
\text { Functional Reach Test. }\end{array}$ & & & \\
\hline Paiva et al. (2012) & $\begin{array}{l}\text { Constituída de } 30 \text { idosos de } \\
\text { ambos os sexos acima de } \\
60 \text { anos de idade que são } \\
\text { praticantes do treinamento } \\
\text { resistido. }\end{array}$ & $\begin{array}{l}\text { Nenhuma intervenção, } \\
\text { apenas aplicação de um } \\
\text { questionário contendo } 10 \\
\text { questões ao total de } 30 \\
\text { idosos acima de } 60 \text { anos, } \\
\text { referentes aos benefícios } \\
\text { que o treinamento resistido } \\
\text { pode trazer dentro das } \\
\text { academias. }\end{array}$ & $\begin{array}{l}\text { Em todas as questões a } \\
\text { porcentagem de respostas } \\
\text { "não", não foram significativas. } \\
\text { A maioria da amostra mostrou } \\
\text { ter desenvolvido várias } \\
\text { qualidades depois de terem } \\
\text { começado a praticar } \\
\text { musculação, principalmente nas } \\
\text { questões de ganho de força } \\
\text { muscular, prevenção e cura de } \\
\text { doenças. }\end{array}$ & $\begin{array}{l}\text { Na primeira questão onde } \\
\text { perguntava se a prática de } \\
\text { musculação trouxe algum } \\
\text { benefício, } 100 \% \text { dos } \\
\text { participantes. na segunda } \\
\text { questão os participantes } \\
\text { praticaram algum outro } \\
\text { treino de força específico } \\
\text { sem ser musculação. } \\
\text { Sendo que } 87 \% \text { dos } \\
\text { participantes } \\
\text { responderam que não e } \\
13 \% \text { responderam que } \\
\text { sim. }\end{array}$ & $\begin{array}{l}\text { Os resultados demonstraram } \\
\text { que a maioria dos idosos que } \\
\text { praticam treinamento } \\
\text { resistido, melhoraram em } \\
\text { questões de força, estética, } \\
\text { doenças e com tudo, as } \\
\text { percepções dos benefícios } \\
\text { que o mesmo pode trazer. }\end{array}$ & $\begin{array}{l}\text { Conclui-se que o treinamento } \\
\text { resistido em idosos pode sim } \\
\text { trazer diversos benefícios, } \\
\text { melhorando a funcionalidade } \\
\text { do idoso e sua qualidade de } \\
\text { vida. }\end{array}$ \\
\hline Moura (2012) & $\begin{array}{l}\text { Participaram } 60 \text { idosos } \\
\text { institucionalizados, } 30 \text { que } \\
\text { realizavam fisioterapia } \\
(\mathrm{GF}) \text { e } 30 \text { que não } \\
\text { realizavam (GNF). }\end{array}$ & $\begin{array}{l}\text { Os instrumentos utilizados } \\
\text { foram: Escala de Equilíbrio } \\
\text { de Berg (EEB), Testes de } \\
\text { Tinetti, Timed Up \& Go } \\
\text { (TUG) e de Alcance } \\
\text { Funcional Anterior (TAF), } \\
\text { e Questionário } \\
\text { Internacional de Atividade } \\
\text { Física (IPAQ). }\end{array}$ & $\begin{array}{l}\text { Referentes a fisioterapia do GF: } \\
\text { frequência semanal, os motivos } \\
\text { pelos quais fazem fisioterapia e } \\
\text { as atividades realizadas durante } \\
\text { as sessões. os idosos do GNF } \\
\text { não realizavam fisioterapia pelo } \\
\text { fato das instituições } \\
\text { participantes da pesquisa } \\
\text { priorizarem o atendimento } \\
\text { fisioterapêutico aos idosos mais } \\
\text { dependentes uma vez que não } \\
\text { havia fisioterapeutas suficientes } \\
\text { nos locais para atender a toda a } \\
\text { demanda das Instituições de } \\
\text { Longa Permanência (ILPI) as as } \\
\text { mesmas não possuíam } \\
\text { condições financeiras para } \\
\text { contratar mais profissionais. }\end{array}$ & $\begin{array}{l}\text { Os testes foram } \\
\text { realizados entre } 10 \text { e } 20 \\
\text { segundos. A pontuação } \\
\text { máxima é de } 12 \text { pontos } \\
\text { para a marcha, de } 16 \text { para } \\
\text { o equilíbrio do corpo e de } \\
28 \text { para a total. }\end{array}$ & $\begin{array}{l}\text { Foi observada diferença no } \\
\text { TUG }(p=0,01) \text {, sendo que o } \\
\text { GF levou mais tempo para } \\
\text { realizar o teste }(19,5 \pm 6,1 \\
\text { segundos) que o GNF }(16,4 \pm \\
7,3 \text { segundos). Também foi } \\
\text { observada diferença no TAF } \\
\text { ( } \mathrm{p}=0,003) \text {, com melhor } \\
\text { desempenho durante o teste } \\
\text { no GNF }(27,6 \pm 6,2 \mathrm{~cm}) \text { que } \\
\text { no GF }(22,4 \pm 5,1 \mathrm{~cm}) .\end{array}$ & $\begin{array}{l}\text { Foi observado no estudo que } \\
\text { idosos que realizavam } \\
\text { fisioterapia apresentaram } \\
\text { menor mobilidade funcional, } \\
\text { menor alcance funcional } \\
\text { anterior e menores níveis de } \\
\text { atividade física. }\end{array}$ \\
\hline Helrigle et al. (2013) & 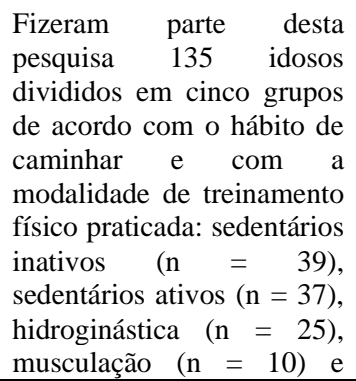 & $\begin{array}{l}\text { Pelo menos duas sessões } \\
\text { semanais de atividade física } \\
\text { estruturada, com duração } \\
\text { de aproximadamente } 50 \\
\text { minutos e dirigidas para } \\
\text { vários componentes de } \\
\text { aptidão física (treino de } \\
\text { resistência aeróbia, força } \\
\text { muscular e flexibilidade). }\end{array}$ & $\begin{array}{l}\text { Os idosos sedentários inativos e } \\
\text { ativos atingiram valores de } \\
48,89 \pm 0,87 \text { e } 52,45 \pm 0,47 \text {, } \\
\text { respectivamente na Escala de } \\
\text { Equilíbrio de Berg (EEB). Os } \\
\text { idosos treinados praticantes de } \\
\text { hidroginástica obtiveram escore } \\
\text { de } 54,04 \pm 0,33 \text {, os praticantes } \\
\text { de musculação } 53 \pm 0,52 \text { e os } \\
\text { praticantes de caminhada de }\end{array}$ & $\begin{array}{l}\text { Os idosos ativos tinham o } \\
\text { hábito de caminhar fora } \\
\text { de casa por mais de } \\
\text { quatro quarteirões, pelo } \\
\text { menos três vezes } \\
\text { semanais, nos seis meses } \\
\text { prévios ao estudo. }\end{array}$ & $\begin{array}{l}\text { Os resultados mostraram que } \\
125 \text { idosos apresentaram } \\
\text { valores da Escala de } \\
\text { Equilíbrio de Berg (EEB) } \\
\text { maiores que 45, 8 idosos } \\
\text { apresentaram valores } \\
\text { menores ou iguais a } 45 \text { e } \\
\text { somente } 2 \text { obtiveram valores } \\
\text { menores ou iguais a } 36 .\end{array}$ & $\begin{array}{l}\text { Observou-se que tanto o } \\
\text { hábito de caminhar quanto a } \\
\text { prática regular da caminhada, } \\
\text { da musculação e da } \\
\text { hidroginástica por mais de } \\
\text { seis meses, aumentam o } \\
\text { equilíbrio funcional dos } \\
\text { idosos. }\end{array}$ \\
\hline
\end{tabular}




\begin{tabular}{|c|c|c|c|c|c|c|}
\hline & caminhada $(n=24)$ & & $53,45 \pm 0,51$ & & & \\
\hline Leenders (2013) & $\begin{array}{l}\text { Composta por } 114 \text { idosos, } \\
\text { separados em dois grupos: } \\
\text { GFA: } 71 \text { idosos, sendo } 14 \\
(19,7 \%) \text { do sexo masculino } \\
\text { e } 57(80,3 \%) \text { do sexo } \\
\text { feminino, com média de } \\
\text { idade de } 73,55 \pm 6,93 \text { anos; } \\
\text { GTR: } 43 \text { idosos, sendo } 27 \\
(62,8 \%) \text { do sexo masculino } \\
\text { e } 16 \text { (37,2\%) do sexo } \\
\text { feminino, com idade média } \\
\text { de } 66,72 \pm 5,26 \text {. }\end{array}$ & $\begin{array}{l}\text { Nenhuma intervenção, } \\
\text { apenas avaliação da } \\
\text { capacidade funcional. } \\
\text { Questionário estruturado } \\
\text { fechado, teste de sentar e } \\
\text { levantar, teste de } \\
\text { dinamometria de força de } \\
\text { preensão manual, Escore de } \\
\text { Lawton, Escala de Katz e } \\
\text { Timed Up \& Go Test } \\
\text { (TUG). }\end{array}$ & $\begin{array}{l}\text { Foram encontradas diferenças } \\
\text { significativas entre os grupos } \\
\text { em relação ao sexo, estado } \\
\text { civil, renda e escolaridade } \\
(p<0,001) \text { O grupo GTR } \\
\text { apresentou média menor no } \\
\text { tempo de deslocamento no } \\
\text { TUG test }(6,24 \pm 0,86 \\
\text { segundos }) \text { em relação ao GFA, } \\
\text { que apresentou média de } 11,24 \\
\pm 4,26 \text { segundos }(p=0,035) .\end{array}$ & $\begin{array}{l}\text { Os exercícios eram } \\
\text { realizados em } 3 \text { séries de } \\
8 \text { a } 12 \text { repetições } \\
\text { máximas, com intervalos } \\
\text { de } 1 \text { minuto entre as } \\
\text { séries, de forma alternada } \\
\text { por segmentos } \\
\text { musculares, adotando-se } \\
\text { a seguinte ordem para a } \\
\text { execução dos exercícios: } \\
\text { remada sentada, extensão } \\
\text { de joelhos, voador, flexão } \\
\text { de joelhos, rosca direta } \\
\text { com halteres, cadeira } \\
\text { abdutora, rosca tríceps, } \\
\text { cadeira adutora; } \\
\text { apresentando incremento } \\
\text { de } 10 \% \text { na carga total a } \\
\text { cada } 4 \text { semanas. }\end{array}$ & $\begin{array}{l}\text { Após serem realizados os } \\
\text { ajustes estatísticos, não foram } \\
\text { encontradas diferenças } \\
\text { estatisticamente significativas } \\
\text { entre os grupos em relação ao } \\
\text { Escore de Lawton, à força de } \\
\text { preensão manual e no teste de } \\
\text { sentar e levantar. }\end{array}$ & $\begin{array}{l}\text { Concluiu-se que idosos } \\
\text { praticantes do treinamento } \\
\text { resistido (TR) apresentaram } \\
\text { desempenho } \\
\text { significativamente melhor no } \\
\text { TUG test, o que está } \\
\text { diretamente relacionado com } \\
\text { a prevenção de quedas e } \\
\text { fraturas. }\end{array}$ \\
\hline $\begin{array}{l}\text { Garatachea et al. } \\
\text { (2014) }\end{array}$ & $\begin{array}{l}\text { Constituída por oito } \\
\text { indivíduos entre } 62 \text { e } 79 \\
\text { anos, sendo quatro homens } \\
\text { e quatro mulheres, } \\
\text { residentes na cidade de Itu - } \\
\text { SP. }\end{array}$ & $\begin{array}{l}\text { Testes utilizados: carga } \\
\text { máxima no exercício } \\
\text { supino reto, quando os } \\
\text { voluntários realizaram e } \\
\text { sustentaram um movimento } \\
\text { com a carga máxima } \\
\text { suportada; o teste de } \\
\text { agachamento com o próprio } \\
\text { peso corporal, em que os } \\
\text { mesmos agacharam e } \\
\text { levantaram durante um } \\
\text { minuto ou até não } \\
\text { conseguirem mais realizar } \\
\text { o movimento completo; e } \\
\text { também o teste de } \\
\text { composição corporal e } \\
\text { antropometria. }\end{array}$ & $\begin{array}{l}\text { Nenhum dos oitos participantes } \\
\text { pediu para interromper, passou } \\
\text { mal ou executou o exercício em } \\
\text { questão equivocado } \\
\text { necessitando interromper o } \\
\text { exercício citado. }\end{array}$ & $\begin{array}{l}\text { A frequência semanal } \\
\text { estabelecida de } \\
\text { treinamento foi de três } \\
\text { sessões por semana, em } \\
\text { dias alternados e durante } \\
\text { o período de dois meses, } \\
\text { totalizando 24 sessões de } \\
\text { treinamento com } \\
\text { exercícios compostos de } \\
\text { três séries de oito a doze } \\
\text { repetições máximas. }\end{array}$ & $\begin{array}{l}\text { Os testes aplicados no estudo } \\
\text { mostraram melhora } \\
\text { expressiva na performance ou } \\
\text { desempenho dos avaliados, } \\
\text { nos testes de resistência de } \\
\text { força (agachamento) e no } \\
\text { teste de 1RM frente ao } \\
\text { momento pré e pós. }\end{array}$ & $\begin{array}{l}\text { Os valores notados na } \\
\text { população estudada } \\
\text { apontaram que este método } \\
\text { de treinamento pode ser } \\
\text { utilizado como uma estratégia } \\
\text { e opção para prevenção da } \\
\text { sarcopenia e melhora da } \\
\text { qualidade de vida e } \\
\text { autonomia, proporcionando } \\
\text { benefícios à saúde dos seus } \\
\text { praticantes. }\end{array}$ \\
\hline Mendes (2014) & 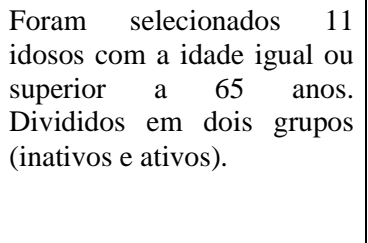 & $\begin{array}{l}\text { Os idosos foram avaliados } \\
\text { pelos testes de capacidade } \\
\text { funcional e submetidos a } \\
\text { treinamento da força. }\end{array}$ & $\begin{array}{l}\text { Observou-se, no presente estudo, } \\
\text { redução no tempo de execução } \\
\text { no teste de caminhar tanto ativo } \\
\text { quanto para inativo. }\end{array}$ & 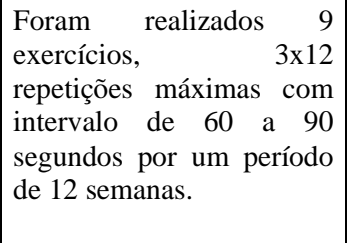 & $\begin{array}{l}\text { As investigações científicas } \\
\text { vêm mostrando que os ativos } \\
\text { tendem a ter maiores ganhos } \\
\text { na flexibilidade e coordenação } \\
\text { motora com treinamento de } \\
\text { força (TF) em idosos, quando } \\
\text { comparados aos inativos. }\end{array}$ & $\begin{array}{l}\text { Observou-se que o programa } \\
\text { de treinamento de força por } \\
\text { um curto período melhora o } \\
\text { desempenho das atividades da } \\
\text { vida diárias dos idosos. }\end{array}$ \\
\hline
\end{tabular}




\begin{tabular}{|c|c|c|c|c|c|c|}
\hline Vechin et al. (2014) & $\begin{array}{l}\text { Participaram } 23 \text { idosos } 14 \\
\text { homens e } 9 \text { mulheres (idade: } \\
64,04 \pm 3,81 \text { anos; peso: } \\
72,55 \pm 16,52 \mathrm{~kg} \text {; altura: } 163 \\
\pm 11 \mathrm{~cm} \text { ). }\end{array}$ & $\begin{array}{l}\text { Os sujeitos realizaram } 2 \\
\text { sessões de familiarização } \\
\text { para se familiarizarem com } \\
\text { os procedimentos de teste de } \\
\text { 1RM do leg press antes do } \\
\text { teste. O teste de } 1 \text { RM foi } \\
\text { realizado } 48 \text { horas após a } \\
\text { última sessão } \\
\text { familiarização. }\end{array}$ & $\begin{array}{l}\text { Ambos os métodos foram } \\
\text { igualmente eficazes na indução } \\
\text { de aumento na área transversal } \\
\text { do quadríceps (CSA) do } \\
\text { quadríceps em uma coorte de } \\
\text { indivíduos mais velhos. No } \\
\text { entanto, diferenças relevantes } \\
\text { nos ganhos de força foram } \\
\text { observadas entre os } 2 \text { grupos de } \\
\text { treinamento (LRT-BFR: } \sim 17 \% \text {; } \\
\text { HRT: } \sim 54 \%) \text {. }\end{array}$ & 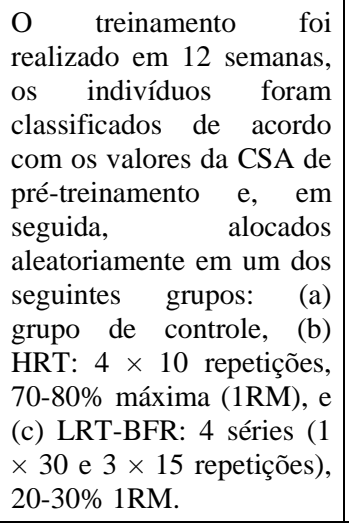 & $\begin{array}{l}\text { Ambos os regimes de } \\
\text { treinamento foram eficazes em } \\
\text { aumentar 1RM pré e pós- } \\
\text { treinamento de leg press } \\
\text { (HRT: } \sim 54 \%, p<0,001 \text {; LRT- } \\
\text { BFR: } \sim 17 \%, \text { p }=0,067 \text { ) e } \\
\text { quadríceps CSA (HRT: 7,9\%, } \\
\text { p < 0,001; LRT BFR: } 6,6 \%, p \\
<0,001) \text {; entretanto, a TRH } \\
\text { parece induzir maiores ganhos } \\
\text { de força. }\end{array}$ & $\begin{array}{l}\text { Em resumo, LRT-BFR } \\
\text { constitui uma abordagem } \\
\text { substituta importante para } \\
\text { HRT como um método de } \\
\text { treinamento eficaz para induzir } \\
\text { ganhos de força e massa } \\
\text { muscular em idosos. }\end{array}$ \\
\hline Dias et al. (2015) & $\begin{array}{l}\text { Vinte e seis mulheres idosas } \\
\text { saudáveis (idade }=67 \pm 6 \\
\text { anos) foram aleatoriamente } \\
\text { designadas para um grupo de } \\
\text { treinamento com foco } \\
\text { excêntrico (ETG; } \mathrm{n}=13 \text { ) ou } \\
\text { um grupo de treinamento } \\
\text { convencional }(\mathrm{CTG} ; \mathrm{n}=13) .\end{array}$ & $\begin{array}{l}\text { Os sujeitos realizaram testes } \\
\text { específicos } \text { (teste r de } \\
\text { caminhada de } 6 \mathrm{~m} \text {, teste } \\
\text { cronometrado de } \text { Up-and- } \\
\text { Go, escada teste de escalada } \\
\text { e teste de elevação da } \\
\text { cadeira). }\end{array}$ & $\begin{array}{l}\text { Ambos os grupos melhoraram a } \\
\text { extensão do joelho } 1 \text { RM }(24- \\
26 \% ; \mathrm{p}=0,021) \text {, teste Up-and- } \\
\text { Go cronometrado }(11-16 \% ; p< \\
0,001) \text {, teste de caminhada de } 6 \\
\mathrm{~m}(9-12 \% ; \mathrm{p}=0,004) \text {, teste de } \\
\text { subir escadas }(8-13 \% ; \mathrm{p}= \\
0,007) \text { e teste de subir escadas } \\
(15-16 \% ; \mathrm{p}<0,001) \text {, mas não } \\
\text { houve diferença significativa } \\
\text { entre os grupos. }\end{array}$ & $\begin{array}{l}\text { Foram realizados } 12 \\
\text { semanas de treinamento de } \\
\text { resistência duas vezes por } \\
\text { semana. Para o ETG, as } \\
\text { fases concêntricas e } \\
\text { excêntricas foram } \\
\text { realizadas com } 1,5 \text { e } 4,5 \mathrm{~s} \text {, } \\
\text { respectivamente, enquanto } \\
\text { para o CTG, cada fase } \\
\text { durante } 1,5 \mathrm{~s} \text {. }\end{array}$ & $\begin{array}{l}\text { A carga usada pelos grupos de } \\
\text { treinamento convencional } \\
\text { com foco excêntrico foi } \\
\text { mesma durante todo } \\
\text { programa de treinamento. } \\
\text { única diferença ocorre no } \\
\text { tempo de exposição à } \\
\text { contração excêntrica: } 1,5 \text { s } \\
\text { para o grupo convencional e } \\
4,5 \text { s para o grupo excêntrico. }\end{array}$ & $\begin{array}{l}\text { A estratégia de aumentar o } \\
\text { tempo de exposição na fase } \\
\text { excêntrica do movimento com } \\
\text { o mesmo volume e intensidade } \\
\text { de treinamento não promove } \\
\text { diferentes adaptações na força } \\
\text { ou capacidade funcional em } \\
\text { comparação ao treinamento } \\
\text { resistido convencional em } \\
\text { mulheres idosas. }\end{array}$ \\
\hline Carvalho et al. (2017) & $\begin{array}{l}\text { Participaram do estudo } 383 \\
\text { sujeitos }(73,2 \pm 8,93 \text { anos }) \\
\text { sendo } 258(67,4 \%) \text { do sexo } \\
\text { feminino e } 125(32,5 \%) \text { do } \\
\text { sexo masculino. }\end{array}$ & \begin{tabular}{|lrrr|} 
Utilizou-se & \multicolumn{2}{r}{ questionário } \\
estruturado & com questões \\
objetivas & e & subjetivas & em \\
relação & a & prática & de \\
exercício. físico. &
\end{tabular} & $\begin{array}{l}\text { Dos } 25,2 \% \text { que praticam } \\
\text { exercício, } 48,5 \% \text { receberam } \\
\text { aconselhamento para prática } \\
\text { através de médicos. Dentre as } \\
\text { dificuldades encontradas, } 31,9 \% \\
\text { dos idosos, a falta de interesse } \\
\text { predomina entre os motivos mais } \\
\text { relatados. }\end{array}$ & $\begin{array}{l}\text { A caminhada era realizada } \\
\text { com frequência de } 3 \text { a } 6 \\
\text { vezes por semana }(59,6 \% ; \\
\mathrm{n}=34) \text { e com duração de } \\
20 \text { a } 30 \text { minutos por sessão } \\
(52,6 \% ; \mathrm{n}=30) \text {. } \\
\text { Questionário: bem } \\
\text { estruturado contendo } 18 \\
\text { questões objetivas e } \\
\text { subjetivas à identificação, } \\
\text { como: idades, sexo, estado } \\
\text { civil, renda familiar, } \\
\text { escolaridade e prática de } \\
\text { exercício físico, tempo de } \\
\text { prática e acompanhamento } \\
\text { profissional. }\end{array}$ & $\begin{array}{l}\text { Os resultados apontaram que } \\
74,8 \% \text { dos idosos não praticam } \\
\text { nenhum tipo de exercício } \\
\text { físico. No entanto, } 90,4 \%(\mathrm{n}= \\
348) \text { consideram importante a } \\
\text { prática de exercícios físicos, } \\
\text { sendo a caminhada o exercício } \\
\text { físico mais praticado. }\end{array}$ & $\begin{array}{l}\text { Sendo assim, programas de } \\
\text { atividade física em Unidades } \\
\text { de Saúde da Família devem ser } \\
\text { implementados como forma de } \\
\text { garantia de acesso e incentivo } \\
\text { para esse grupo etário. }\end{array}$ \\
\hline
\end{tabular}


Os resultados encontrados neste estudo evidenciaram que idosos praticantes de treinamento de força tiveram efeito positivo, como: melhora da qualidade de vida, redução da perda de massa óssea, aumento da força muscular, aumento da capacidade e mobilidade funcional. Geralmente os idosos, perdem força muscular à medida que envelhecem. Além da redução da força muscular, e o comprometimento avançado da mobilidade com a idade certamente acarretam redução da atividade de vida diária (Srikanthan, Hevener e Karlamangla, 2010).

É relevante compreender que à medida que a idade aumenta os idosos se tornam menos ativos, sua capacidade física diminui e por conta disso muitos idosos têm medo de envelhecer tornando-se dependentes de suas limitações. Logo, não surpreende que o treinamento de força contribua para um resultado positivo na função física em idosos, independentemente da hipertrofia muscular (Tieland, Trouwborst e Clark, 2018). Os estudos com pessoas idosas têm documentado a influência dos efeitos do treinamento de força para melhorar a qualidade de vida por meio do alívio de dores articulares, maior independência funcional e melhora da autoestima (Mendes, 2014).

A segurança musculoesquelética e segurança cardiovascular do treinamento de força também têm sido evidenciadas, mesmo diante de co-morbidades. No momento atual, um significativo corpo de evidências justifica a utilização do treinamento de força para promoção de saúde, terapêutica e reabilitação (Câmara, 2012). Importante destacar os princípios básicos para as aplicações do treinamento de força, uma seleção adequada dos exercícios deve ocorrer na prescrição e planejamento dos programas de tratamento, evitando-se movimentos que possam ser inadequados para cada situação.

Os benefícios que os idosos adquirem com o exercício resistido vão além da estrutura física. Por exemplo, Granacher et al. (2013) indicam que a execução regular do treinamento de força está relacionada ao aumento da longevidade, à prevenção da perda cognitiva, à diminuição da frequência de quedas e ocorrências de fraturas, mesmo que sua prática tenha início após a sexta década de vida. Tal indício pode ter um efeito positivo no equilíbrio e marcha dos idosos, o que resulta na prevenção de quedas e fraturas (Civinski; Montibeller; Braz, 2011). Logo o exercício com uso de uma resistência contribui para tornar lento e até mesmo reverter à perda da mobilidade funcional, regresso da perda de massa óssea e força muscular. A realização frequente de exercícios físicos, principalmente o treinamento de força, é um dos pontos fulcrais da manutenção adequada da função física em idosos, sendo considerada medida preventiva para as mudanças musculares que se dão no processo do envelhecimento, visto que suas melhoras e efeitos aplicam-se à maioria das pessoas, independentemente do seu estado de saúde (Finger et al., 2014; Vechin et al. 2014).

Poder-se-ia dizer aqui que a nova geração de idosos envelhece hoje com mais consciência, com mais autonomia e expectativa de um futuro mais saudável. Acredita-se que o estilo de vida ativo é de suma importância para melhoria da qualidade de vida, além de restabelecer a saúde no decorrer do processo de envelhecimento (Corrêa; Borba-Pinheiro; Dantas, 2013). Aproximadamente entre 20 e 30\% dos indivíduos com 65 anos ou mais apresentam algum grau de inabilidade funcional, a qual é definida como falha ou dependência na execução de certos gestos e de certas funções rotineiras (Orsatti et al., 2011; Peterson e Gordon, 2011).

Por esta razão, este trabalho tem o objetivo de defender a necessidade e o efeito do treinamento de força na capacidade funcional do idoso. Este exercício atua de forma benéfica no sistema musculoesquelético e composição corporal, sendo efetivado progressivamente (Peterson e Gordon, 2011). Um ponto relevante a ser citado com relação aos benefícios que o treinamento de força proporciona aos idosos, se dá em relação à associação do aumento de força muscular favorecido pelo treino, alteração da mobilidade funcional e equilíbrio, assim como redução expressiva no número de quedas em idosos (Araújo, Fló e Muchale, 2010; Cakar et al., 2010). Também com resultados semelhantes, estudos indicam que o treinamento de força são fontes de proteção para a preservação da mobilidade, marcha, equilíbrio e consequentemente, diminuição dos riscos de quedas, fraturas, inabilidade funcional, internações e mesmo óbitos (Abellán et al., 2011; Virtuoso Júnior e Guerra, 2011). 
Resultados demonstraram melhora da resistência muscular e da funcionalidade nos idosos após um treino de exercício resistido (Dias et al., 2015).

Sendo assim, fica evidente que o exercício resistido contribui positivamente na vida do idoso ativo, pois o treino resistido é eficaz para melhora da densidade óssea, equilíbrio, postura, potência, força muscular e consequentemente melhora da mobilidade funcional.

\section{Considerações Finais}

A presente revisão identificou que o treinamento de força em idosos ao longo de 15 semanas, entre duas ou três vezes semanais com duração aproximada de 1 hora, é capaz de promover melhora da capacidade funcional com a sugestão de aumento da massa muscular, melhora da força muscular, aumento ou manutenção da densidade mineral óssea, melhora do equilíbrio, melhora da marcha, progresso da mobilidade funcional e independência de idosos. Todos esses fatores possibilitam ao idoso melhor execução de suas atividades diárias. Assim, sugere-se aqui que os profissionais de saúde incentivem aos idosos incluírem em suas atividades físicas o treinamento de força com o propósito de restabelecer a saúde no decorrer do envelhecimento. Assim como, sugere-se que novas pesquisas sejam conduzidas pesquisando essa tela em questão a fim de trazer informações importantes no processo de envelhecimento.

\section{Agradecimentos}

Os autores agradecem a participação de todos os voluntários dessa pesquisa.

\section{Referências}

Abellán, A.; Esparza, C.; Castejón, P.; Pérez J. (2011). Epidemiología de la discapacidad y la dependencia de la vejez en España. Gaceta Sanitaria, 25 (2), 5 11.

Agner, V. F. C.; Gomes, A. R. S.; Paz, L. P.; Correa, C. L. (2013). Identificação do perfil físico-funcional de idosos de uma instituição de longa permanência. Rev Pesq Fisio, 3 (3), 152-167.

American College of Sports Medicine position stand (2011). Quantity and quality of exercise for developing and maintaining cardiorespiratory, musculoskeletal, and neuromotor fitness in apparently healthy adults: guidance for prescribing exercise. Med Sci Sports Exerc, 43 (7), 1334-1359.

Araújo, M. L. M.; Fló, C. M.; Muchale, S. M. (2010). Efeitos dos exercícios resistidos sobre o equilíbrio e a funcionalidade de idosos saudáveis. Fisioter Pesq, 17 (3), 277-283.

Balzi, D.; Lauretani, F.; Barchielli, A.; Ferrucci, L.; Bandinelli, S.; Buiatti, E.; Milaneschi, Y.; Guralnik, J. M. (2010). Risk for disability in older persons over 3-years follow-up. Age Ageing, 39 (1), 92-98.

Carvalho, D. A.; Brito, A. F.; Santos, M. A. P.; Nogueira, F. R. S.; Sá, G. G. M.; Oliveira Neto, J. G.; Martins, M. C. C.; Santos, E. P. (2017). Prevalência da prática de exercícios físicos em idosos e sua relação com as dificuldades e a falta de aconselhamento profissional específico. Rev Bras Ciênc e Mov, 25 (1), 2940.

Cakar, E.; Dincer, U.; Kiralp, N. Z.; Cakar, D. B.; Durmus, O.; Kilac, H.; Soydan, F. C.; Sevinc, A.; Alper, C. (2010). Jumping combined exercise programs reduce fall risk and improve balance and life quality of elderly people who live in a long-term care facility. Eur J Phys Rehabil Med, 46 (1). 59-67.

Câmara, L. C.; Bastos, C. C.; Volper, E. F. T. (2012). Exercício resistido em idosos frágeis: uma revisão da literatura. Fisioter Mov, 25 (2), $435-443$.

Cipriani, N. C. S.; Meurer, S. T.; Benedetti, T. R. B.; Lopes, M. A. (2010). Aptidão física funcional de idosas praticantes de atividades físicas. Rev Bras Cineantropom Desempenho Hum, 12 (2), 106-111.

Civinski. C.; Montibeller, A.; Braz, A. L. O. (2011). A importância do exercício físico no envelhecimento. Rev da Unifebe, 9 (1), $163-75$.

Correa, D. G.; Borba-Pinheiro, C. J.; Dantas, E. H. M. (2013). Qualidade de vida no envelhecimento humano. Praxia, 1 (1), 37-52.

Dias, C. P.; Toscan, R.; de Camargo, M.; Pereira, E. P.; Griebler, N.; Baroni, B. M.; Tiggemann, C. L. (2015). Effects of eccentric-focused and conventional resistance training on strength and functional capacity of older adults. Age (Dordr), 37 (5), 2015.

Estrela, C. (2018). Metodologia Científica: Ciência, Ensino, Pesquisa. Editora Artes Médicas.

Elias, R. G. M.; Gonçalves, E. C. D. A.; Moraes, A. C. F. D.; Moreira, C. F.; Fernandes, C. A. M. (2012). Aptidão física funcional de idosos praticantes de hidroginástica. Rev Bras Geriatr Gerontol, 15 (1), 79-86. 
Finger, D.; Goltz, F. R.; Umpierre, D.; Meyer, E.; Rosa, L. H.; Schneider, C. D. (2014). Effects of protein supplementation in older adults undergoing resistance training: A systematic review and meta-analysis. Sport Med, 45 (2), 245-55.

Granacher, U.; Lacroix, A.; Muehlbauer, T.; Roettger, K.; Gollhofer, A. (2013). Effects of Core Instability Strength Training on Trunk Muscle Strength, Spinal Mobility, Dynamic Balance and Functional Mobility in Older Adults. Gerontology, 59 (2), $105-113$.

Garatachea, N.; Pareja-Galeano, H.; Sanchis-Gomar, F.; Santos-Lozano, A.; FiuzaLuces, C.; Morán, M.; Emanuele, E.; Joyner, M. J.; Lucia, A. (2015). Exercises attenuates the major hallmarks of aging. Rejuvenation Res, 18 (1), 57-89.

Helrigle, C.; Ferri, L. P.; Netta, C. P. O.; Belem, J. B.; Malysz, T. (2013). Efeitos de diferentes modalidades de treinamento físico e do hábito de caminhar sobre o equilíbrio funcional de idosos. Fisioterapia Mov, 26 (2), 321-327.

Leenders, M.; Verdijk, L. B.; Van Der Hoeven, L.; Van Kranenburg, J.; Nilwik, R.; Van Loon, L. J. (2013). Elderly Men and Women benefit Equally from Prolonged Resistance-Type Exercise Training. J Gerontol A Biol Sci Med Sci, 68 (7), 769- 779.

Lima, M. P. (2014). Envelhecimento(s). $1^{\mathrm{a}}$ ed. Coimbra: Imprensa da Universidade de Coimbra, p. 141.

Liu, C. J.; Latham, N. (2011). Can progressive resistance strength training reduce physical disability in older adults? A meta-analysis study. Disabil Rehabil, 33 (2), 87-97.

Marques, E. A.; Mota, J.; Carvalho, J. (2012). Exercise effects on bone mineral density in older adults: a meta-analysis of randomized controlled trials. Age (Dordr), 34 (6), 1493-1515.

Mendes, G. D. S. O. (2014). A influência do treinamento resistido de força sobre as capacidades funcionais de indivíduos idosos: uma revisão sistemática. Monografia de Graduação. UCB-BSB. Brasília.

Moreira, M. J. G.; Henriques, F. C. (2014). Envelhecimento e Saúde: prioridades políticas num Portugal em mudança. $1^{\text {a }}$ ed. Lisboa: Instituto Hidrográfico, p. 295.

Moura, M. S.; Pedrosa, M. A. C.; Costa, E. L.; Bastos Filho, P. S. C.; Sayão, L. B., Sousa, T. S. (2012). Efeitos de exercícios resistidos, de equilíbrio e alongamentos sobre a mobilidade funcional de idosas com baixa massa óssea. Rev Bras Ativ Fis e Saúde, 17 (6), 2012.

Oliveira, L. P. B. A.; Menezes, M. P. (2011). Representações de fragilidade para idosos no contexto da estratégia saúde da família. Enferm, 20 (2), $301-309$.

Orsatti, F. L.; Dalanesi, R. C.; Maestá, N.; Náhas, E. A. P.; Burini, R. C. (2011). Redução da forma muscular está relacionada à perda muscular em mulheres acima de 40 anos. Rev Bras Cineantropom Desempenho Hum, 13 (1), 36-42.

Peterson, M. D.; Gordon, P. M. (2011). Resistance exercise for the aging adult: clinical implications and prescription guidelines. Am J Med, 124 (3), 194-198.

Paiva, T. D.; Santos, C. F.; Silva, E. M.; Silva, E. R.; Silva, K. R.; Borragine, S. O. F. (2012). Exercício resistido e a saúde do idoso. Lect Educación Fís Deportes, 17 (6), 1-1.

Rejeski, W. J.; Ip, E. H.; Bertoni, A. G.; Bray, G. A.; Evans, G.; Gregg, E. W.; Zhang, Q. (2012). Lifestyle change and mobility in obese adults with type 2 diabetes. N Engl J Med, 366 (13), 1209-1217.

Rodrigues, D. S. (2014). Atividade física na prevenção e no controle dos fatores de risco para as doenças cardiovasculares. Monografia de Graduação. UEPPB. Paraíba.

Silva, A. C. S.; Santos, I. (2010). Promoção do autocuidado de idosos para o envelhecer saudável: aplicação da teoria de Nola Pender. Enferm, 19 (4). 745 753.

Srikanthan, P.; Hevener, A. L.; Karlamangla, A. S. (2010). Sarcopenia exacerbates obesity-associated insulin resistance and dysglycemia: findings from the National Health and Nutrition Examination Survey III. PLoS One 5:e10805. doi: 10.1371/journal.pone.0010805

Tieland, M.; Trouwborst, I.; Clark, B. C. (2018). Skeletal muscle performance and ageing. J Cachexia Sarcopenia Muscle, 9 (1), $3-19$.

Vechin, F. C.; Libardi, C. A.; Conceição, M. S.; Damas, F. R.; Lixandrão, M. E.; Berton, R. P.; Tricoli, V. A. A.; Roschel, H. A.; Cavaglieri, C. R.; ChaconMikahil, M. P. T.; Ugrinowitsch, C. (2014). Comparisons between low-intensity resistance training with blood flow restriction and high-intensity resistance training on quadriceps muscle mass and strength in elderly. J Strength Cond Res, 29 (4), 1071-1076.

Virtuoso Júnior, J. S.; Guerra, R. O. (2011). Incapacidade funcional em mulheres idosas de baixa renda. Cien Saúde Colet, 16 (5), $2541-2548$. 